\title{
Development and Validation of the Expectations of Aesthetic Rhinoplasty Scale
}

\author{
Mohsen Naraghi ${ }^{1,2,3}$, Mohammad Atari ${ }^{4}$ \\ ${ }^{I}$ Division of Rhinology and Facial Plastic Surgery, Department of Otorhinolaryngology, Head and Neck Surgery, Tehran University of Medical \\ Sciences, Tehran; ${ }^{2}$ Otorhinolaryngology Research Center, Tehran University of Medical Sciences, Tehran; ${ }^{3}$ Rhinology Research Society, \\ Tehran; ${ }^{4}$ Department of Psychology, Faculty of Psychology and Education, University of Tehran, Tehran, Iran
}

Background There is a growing concern in the field of aesthetic surgery about the need to measure patients' expectations preoperatively. The present study was designed to develop and validate the Expectations of Aesthetic Rhinoplasty Scale (EARS), and to compare expectations between rhinoplasty patients with and without body dysmorphic disorder (BDD).

Methods In total, 162 college students and 20 rhinoplasty candidates were recruited. The measures included the newly developed EARS, a measure of psychopathology, and demographics. The DSM-IV structured clinical interview for BDD was used to confirm the diagnosis in rhinoplasty patients.

Results The EARS was constructed of six items based on their significant content validity. In the scale development phase, Cronbach's alpha was 0.87 . The test-retest reliability coefficient of the scale was satisfactory (intraclass correlation coefficient, $0.94 ; 95 \%$ confidence interval, $0.82-0.98)$ over a four-week period. Scores on the EARS were significantly positively correlated with psychopathological symptoms $(r=0.16 ; \mathrm{P}<0.05)$. Moreover, comparison of EARS scores between $\operatorname{BDD}(M=25.90$, standard deviation $[S D]=6.91)$ and non-BDD rhinoplastic patients $(M=15.70, S D=5.27)$ suggested that BDD patients held significantly higher expectations $(\mathrm{P}<0.01)$.

Conclusions The expectations of aesthetic rhinoplasty patients toward surgery may play a crucial role in their postoperative satisfaction. While the value of patients' expectations is clinically recognized, no empirical study has measured these expectations in a psychometrically sound manner. The current study developed and validated the EARS. It may be easily used as a valid and reliable instrument in clinical and research settings.

Keywords Rhinoplasty / Psychology / Psychometrics / Body dysmorphic disorders
Correspondence: Mohammad Atari Department of Psychology, Faculty of Psychology and Education, University of Tehran, No. 2417, Valiasr Avenue, Tehran 1517843318, Iran

Tel: +98-912-636-3263

Fax: +98-218-836-4403

E-mail:mohammad.attari@yahoo. com

This article was presented at the American Academy of Facial Plastic and Reconstructive Surgery (AAFPRS) in September of 2015; Dallas, Texas, US.

No potential conflict of interest relevant to this article was reported.

Received: 7 Jan $2016 \bullet$ Revised: 25 May $2016 \bullet$ Accepted: 21 Jun 2016

pISSN: 2234-6163・ elSSN: 2234-6171・ http://dx.doi.org/10.5999/aps.2016.43.4.365・Arch Plast Surg 2016;43:365-370

\section{INTRODUCTION}

It has been suggested that cosmetic surgery is essentially body image surgery and that facial plastic surgery can particularly enhance body image, quality of life ( $\mathrm{QL})$, personality perceptions, and perceived age. On the other hand, a review of the evidence [1] concluded that it was scientifically premature to assume that cosmetic surgery necessarily leads to direct psychological benefits. There appears to be a general lack of well-controlled research into the range of possible psychological outcomes following cos- 
metic surgery. While patients typically report high rates of satisfaction with surgical results [2,3], long-term satisfaction and associated mental health implications have not been thoroughly researched. A recent study reported a significant decrease in the QoL of rhinoplasty patients [4], suggesting that the decreased QoL could be associated with unnecessary operations, medical errors, and undergoing rhinoplasty solely because of its popularity [5]. Subscribers to a biomedical model of a simple and causal relationship between cosmetic surgical intervention and improved body image have been accused of 'colluding with the myth that QoL necessarily improves when physical appearance is enhanced' [6].

The psychological aspects of aesthetic rhinoplasty have started to attract clinicians' and researchers' attention in the past few decades. Generally, it has been shown that aesthetic rhinoplasty patients show stronger symptoms of psychopathology in comparison with control groups [7] and their self-esteem scores are lower than control populations [8]. Body dysmorphic disorder (BDD) has also attracted much attention from researchers. A high prevalence of BDD has been reported among patients who undergo facial plastic surgical procedures, particularly rhinoplasty [9].

One of the most prevalent psychiatric disorders among aesthetic rhinoplasty patients is BDD. Patients who suffer from $\mathrm{BDD}$ are extremely dissatisfied with their physical appearance. $\mathrm{BDD}$ is defined as preoccupation with an imagined unrealistic defect in one's appearance [10]. Moreover, this preoccupation causes considerable distress or impairment in social, occupational, or other aspects of life. The preoccupation may be focused on any part of the body; however, some parts of the body are more frequently observed as the center of the patient's attention. The nose is a facial component which is frequently targeted in BDD patients [11].

Patients with BDD often apply for consecutive cosmetic surgeries in order to improve their self-perceived attractiveness. A high percentage of patients with BDD seek cosmetic treatments [12]. It has been suggested that BDD patients hold unrealistic expectations toward cosmetic procedures and are dissatisfied with the outcome postoperatively regardless of the actual surgical outcome [13]. Research suggests that BDD patients need psychiatric/psychological interventions and not cosmetic surgery [14]. Performing a cosmetic operation in such cases may complicate the procedure for the surgeon and, more importantly, does not improve the QoL of the patient [15]. Such patients often hold high expectations of surgery and their postoperative dissatisfaction may be manifested through depression, anxiety, and even physical violence toward the surgeon [16]. The abovementioned issues highlight the importance of identifying patients with BDD and patients with unrealistically high expectations before surgical interventions.

Generally, cosmetic surgery may be considered appropriate solely on psychological grounds, but such surgery could potentially be conducted without the appropriate preoperative psychological assessments and guidance [17]. Cosmetic surgery without prior psychological screening may serve to worsen patients' QoL [18]. Psychological assessments informing the decision to operate are clinically and ethically important, particularly for screening out BDD patients [19]. A recent review [20] noted that between 7 and 15 percent of seekers of cosmetic surgery meet the diagnostic requirements for the presence of BDD. Surgeons are encouraged to ensure that people's expectations of surgery are both rational and realistic.

While expectations of aesthetic rhinoplasty patients are of absolute importance, they have not been studied extensively. Obviously, the expectations of these patients vary greatly and experienced surgeons can confirm the diversity of expectations toward the operation. Some patients primarily focus on the social and interpersonal effects of the surgery, while others may be more obsessed with facial aesthetic issues. What patients generally agree on is the expectation of some degree of improvement in all three dimensions: social, psychological, and aesthetic. This seems even more important since the election of rhinoplasty is primarily influenced by psychological factors rather than facial aesthetic proportions [21].

The purpose of this study was to develop and validate the Expectations of Aesthetic Rhinoplasty Scale (EARS) as a useful preoperative questionnaire for rhinoplasty candidates. The study consists of two sections: development and validation. In the developmental stage, the objective was to construct a brief scale that covered all of the aspects of expectations needed to produce a meaningful score. In the validation process, the criterion validity of the scale was examined. One group of aesthetic rhinoplasty patients with BDD were compared to a matched group of candidates who were not diagnosed with BDD.

\section{METHODS}

\section{Participants}

Ethical approval was obtained from the university's ethics committee. In the scale construction phase, a total sample of 162 college students ( 105 women, 55 men, and 2 participants who did not report their sex) was recruited. The participants came from different fields of study. The mean age of the participants was 24.4 (standard deviation [SD] = 4.41).

In the validation process, 20 patients were recruited using a purposeful sampling method. The purposeful sampling method 


\section{Table 1. The demographic characteristics of ten BDD patients} and ten non-BDD patients

\begin{tabular}{|lcc|}
\hline Characteristic & $\begin{array}{c}\text { BDD rhinoplasty } \\
\text { candidates }\end{array}$ & $\begin{array}{c}\text { Non-BDD rhinoplasty } \\
\text { candidates }\end{array}$ \\
\hline No. & 10 & 10 \\
Age (SD) & $22.80(5.47)$ & $29.90(8.05)$ \\
Sex & & 3 \\
$\quad$ Male & 3 & 7 \\
$\quad$ Female & 7 & 5 \\
Marital status & 2 & 5 \\
$\quad$ Married & 8 & 2 \\
Single & & 8 \\
History of cosmetic surgery & 7 & \\
$\quad$ Yes & 3 & \\
$\quad$ No & $23.32(4.74)$ & \\
Body mass index (SD) & & \\
\hline BDD, body dysmorphic disorder; SD, standard deviation. & \\
\hline
\end{tabular}

is a non-probability technique of sampling that includes participants if they satisfy a researcher-determined criterion. The demographic characteristics of aesthetic rhinoplasty patients in both the BDD group and non-BDD group are presented in Table 1 .

\section{Measures}

Expectations of Aesthetic Rhinoplasty Scale 6 (EARS-6)

An item pool of six items was administered to the participants. The items were declarative statements about aesthetic rhinoplasty. A sample item was "a nose job would improve my quality of life". Response options were provided in a 6-point Likert scale ranging from "completely agree" to "completely disagree".

\section{Symptom Checklist 25 (SCL-25)}

The SCL-25 is a shortened form of the well-known Symptom Checklist 90 (SCL-90). It has been developed by factor analytic approaches and consists of 25 items. Response options represented frequency from "never" to "very much" in a 5-point Likert scale. The score may be interpreted as a general index of psychopathological symptoms. Satisfactory psychometric properties of the SCL-25 have been reported [22]. The Cronbach's alpha coefficient of this scale was 0.93 in this study.

\section{Demographics}

A brief demographic questionnaire was administered to the participants. It consisted of age, sex, educational level, marital status, and socio-economic status (SES).

\section{Reliability assessment}

Reliability of the EARS was assessed by two approaches. The Cronbach's alpha was calculated in order to evaluate the internal consistency, and the test-retest reliability coefficient ensured the temporal stability of the scale over a four-week period.

\section{Validity assessment}

Four types of validity were assessed (i.e., face validity, content validity, criterion validity, and construct validity). Face and content validity were investigated by an independent panel of three experts. Construct validity was assessed using principal component analysis (PCA). Criterion validity was evaluated by a casecontrol study between BDD patients and non-BDD candidates.

\section{Procedure}

Prior to administration, respondents were asked if they had undergone cosmetic rhinoplasty. Those who had not undergone cosmetic rhinoplasty were included and asked to complete the questionnaires. In the first phase, they were told to respond to the EARS as if they had been offered a free rhinoplasty. Moreover, fifteen students completed the EARS two times over a four-week period for test-retest reliability assessment purposes. Informed consent was obtained verbally. In the validation process, ten rhinoplasty patients were diagnosed with BDD by a clinical psychologist. He used the structured clinical interview for DSM-IV (SCID) to diagnose BDD and other psychiatric disorders (e.g., generalized anxiety disorder or major depressive disorder). In addition, ten other aesthetic rhinoplasty candidates who were not diagnosed with BDD formed the control group.

\section{Statistical analysis}

Data gathering and data coding were performed in a blinded manner. Descriptive statistics, bivariate correlation coefficients, the independent t-test, one-way analysis of variance (ANOVA), and PCA were performed using PASW ver. 18 (SPSS Inc., Chicago, IL, USA).

\section{RESUITS}

\section{Reliability}

A Cronbach's alpha of 0.87 was obtained for the scale. Additionally, test-retest reliability over a four-week period in terms of the intraclass correlation coefficient (ICC) was 0.94 ( $95 \%$ confidence interval $=0.82-0.98$ ).

\section{Validity}

Face and content validity were reported to be satisfactory by one otolaryngologist, one plastic surgeon, and one psychologist. Pearson correlation coefficients between the EARS, SCL-25, SES, and educational level are presented in Table 2. 


\section{Table 2. Inter-correlations between the studv's variables}

\begin{tabular}{lcccc|}
\hline Study & $\mathbf{1}$ & $\mathbf{2}$ & $\mathbf{3}$ & $\mathbf{4}$ \\
\hline EARS-6 & 1 & - & - & - \\
SCL-25 & $0.16^{\star}$ & 1 & - & - \\
SES & 0.08 & -0.07 & 1 & - \\
Education & 0.05 & $-0.18^{\star}$ & 0.13 & 1 \\
\hline
\end{tabular}

EARS-6, Expectations of Aesthetic Rhinoplasty Scale 6; SCL-25, Symptom Checklist 25; SES, socio-economic status.

${ }^{*} \mathrm{P}<0.05$.

Moreover, a PCA was performed on the scale. The KaiserMeyer-Olkin measure of sampling adequacy was 0.804 and Bartlett's test of sphericity was significant $(\mathrm{P}<0.001)$. Therefore, the data matrix was factorable. The findings suggested a single-factor solution. The items' characteristics are presented in Table 2. One extracted component accounted for $60.3 \%$ of the total variance.

\section{Socio-demographic differences}

A Pearson correlation, independent t-test, and one-way ANOVA were performed to detect differences. The independent $t-$ test detected no significant difference in scores between men and women $(P>0.05)$. Furthermore, there was no significant difference between participants with different educational qualifications $(P>0.05)$. Yet, those with higher SES had higher expectations toward rhinoplasty $(\mathrm{P}<0.05)$.

\section{Criterion validity assessment}

Two groups of aesthetic rhinoplasty candidates were compared in this stage. The first group had been diagnosed with BDD while the second group was not diagnosed with the disorder. An independent $t$-test was used to compare the EARS scores of the two groups. The results of the comparison are summarized in Table 3.

\section{DISCUSSION}

This study's objective was to develop and validate a brief scale to evaluate expectations of rhinoplasty patients. The motivation for conducting this study was the existing clinical void for a questionnaire to evaluate patients' expectations. Some patients develop unrealistically high expectations of a cosmetic procedure, which may actually result in dissatisfaction with the outcome regardless of the procedure's quality. Surgeons who perform cosmetic operations can consider unrealistic expectations as a potential index of psychological disturbance, as the findings of this study showed.

The findings of this study provided evidence for the reliability and validity of the EARS. The questionnaire was internally consistent and temporally stable. This 6-item scale demonstrated good face and content validity. The PCA suggested that the developed scale captures the one-dimensional expectations toward rhinoplasty. The total score of the EARS was significantly correlated with a measure of psychopathological symptoms, which was consistent with previous research [7].

Clinical experience suggests that patients with significantly higher expectations show more dissatisfaction postoperatively. There is now an opportunity to empirically measure that hypothesis, since the current study developed a reliable and valid measurement tool for assessment of expectations. Moreover, Gorney and Martello [23] suggested avoiding patients who fit the SIMON criteria. SIMON is an acronym standing for Single, Immature, Male, Over-expectant, and Narcissistic. Each of these characteristics may be easily measured except for the "over-expectant" criterion. Marital status, psychological maturity, sex, and narcissistic personality may be assessed by short validated questionnaires; however, valid and reliable measurement of rhinoplasty patients' expectations had not been studied before. Our newly developed measure may also be specifically useful for detecting gender differences in preoperative expectations, considering recent findings concerning gender differences in rhinoplasty candidates $[24,25]$.

Expectations toward cosmetic procedures such as rhinoplasty may lack objectivity. On the other hand, it is possible for a patient to have a crooked nasal shape, without attaching significance to this condition, and consequently, the patient does not show unrealistic expectations when seeking rhinoplasty. Higher expectations may indicate that the patient anticipates psychosocial changes based on the operation (as reflected in the EARS items).

Interestingly, analyses indicated no significant difference between women and men in the EARS scores. This may be explained by the fact that women may be more interested in aesthetic rhinoplasty but they may not necessarily hold higher ex-

Table 3. Comparison of BDD candidates and non-BDD candidates on the EARS

\begin{tabular}{|c|c|c|c|c|c|c|}
\hline Group (no.) & EARS mean & SD & t-test statistic & df & P-value & Cohen's $d$ \\
\hline BDD candidates $(10)$ & 25.90 & 6.91 & 3.71 & 18 & $P<0.01$ & 1.28 \\
\hline Non-BDD candidates (10) & 15.70 & 5.27 & & & & \\
\hline
\end{tabular}

BDD, body dysmorphic disorder; EARS, Expectations of Aesthetic Rhinoplasty Scale; SD, standard deviation; df, degrees of freedom. 
Table 4. Expectations of Aesthetic Rhinoplasty Scale (EARS) items' statistical characteristics

\begin{tabular}{|c|c|c|c|c|c|}
\hline Item no. & Item & Mean (SD) & Loading & $\begin{array}{l}\text { Corrected item-total } \\
\text { correlation }\end{array}$ & $\begin{array}{l}\text { Alpha if } \\
\text { deleted }\end{array}$ \\
\hline 1 & I expect the nose job to make my face more beautiful. & $1.33(1.29)$ & 0.58 & 0.46 & 0.87 \\
\hline 2 & I expect the nose job to improve my quality of life. & $1.11(1.33)$ & 0.80 & 0.67 & 0.84 \\
\hline 3 & I believe nose job surgery can improve my self-esteem significantly. & $1.51(1.51)$ & 0.87 & 0.78 & 0.82 \\
\hline 4 & After the nose job, I will be more attractive. & $1.90(1.64)$ & 0.83 & 0.73 & 0.83 \\
\hline 5 & A nose job could improve my social interactions. & $1.20(1.24)$ & 0.84 & 0.74 & 0.83 \\
\hline 6 & Those who get a nose job are more attractive. & $1.99(1.35)$ & 0.72 & 0.60 & 0.85 \\
\hline
\end{tabular}

pectations. Furthermore, it is recommended that future research compare the EARS scores of male and female rhinoplasty patients in a larger sample. Age, education, and marital status did not play significant roles in the expectations of the patients. In contrast, SES had a positive relationship with expectations. Statistical analysis suggested that participants at higher socio-economic levels held higher expectations toward rhinoplasty.

Twenty aesthetic rhinoplasty candidates were categorized into two groups. The first group had been diagnosed with BDD, and the second group was considered the control group. Those who were diagnosed with BDD had significantly higher expectations toward surgery. These findings supported the criterion validity of the scale. Moreover, those with BDD were significantly more likely to have experience undergoing a previous cosmetic procedure. These findings are consistent with the notion that overly expectant patients may be psychologically disturbed.

High expectations of patients toward rhinoplasty may result in dissatisfaction after surgery. This study developed and validated the EARS to be used in clinical practice and research settings. This scale is in its initial stages of development, and it is highly recommended that the psychometric properties of the scale be studied in larger samples. Further research is required to examine the relationship between the EARS, body image, surgery outcome satisfaction, self-esteem, etc. To sum up, this 6-item self-report scale was found to have adequate psychometric properties.

\section{Clinical implications}

The present study developed a 6-item questionnaire for assessment of patients' expectations prior to rhinoplasty. In clinical practice, surgeons may easily use this questionnaire (Table 4) to screen out highly expectant patients. Completing this brief questionnaire can take only 1 to 2 minutes. Thereafter, overly expectant candidates of rhinoplasty may be referred to a psychologist for further consultation. If psychological consultation is not available, the surgeon can talk with patients to alleviate their expectations. The surgeon can also use preoperative simulation tech- niques to show the prospective result to the expectant patient. Following this simple procedure, surgeons can screen out problematic patients with minimal cost, energy, and time. Realistic preoperative expectations may also contribute to postoperative patient satisfaction.

\section{ORCID}

Mohammad Atari http://orcid.org/0000-0002-4358-7783

\section{REFERENCES}

1. Sarwer DB, Crerand CE. Body image and cosmetic medical treatments. Body Image 2004; 1:99-111.

2. Faria FS, Guthrie E, Bradbury E, et al. Psychosocial outcome and patient satisfaction following breast reduction surgery. Br J Plast Surg 1999;52:448-52.

3. Sarwer DB, Wadden TA, Pertschuk MJ, et al. The psychology of cosmetic surgery: a review and reconceptualization. Clin Psychol Rev 1998;18:1-22.

4. Mohammadshahi M, Pourreza A, Orojlo PH, et al. Rhinoplasty as a medicalized phenomenon: a 25-center survey on quality of life before and after cosmetic rhinoplasty. Aesthetic Plast Surg 2014;38:615-9.

5. Clarke A, Lester KJ, Withey SJ, et al. A funding model for a psychological service to plastic and reconstructive surgery in UK practice. Br J Plast Surg 2005;58:708-13.

6. Rumsey N, Harcourt D. Body image and disfigurement: issues and interventions. Body Image 2004;1:83-97.

7. Naraghi M, Atari M. Comparison of patterns of psychopathology in aesthetic rhinoplasty patients versus functional rhinoplasty patients. Otolaryngol Head Neck Surg 2015;152: 244-9.

8. Naraghi M, Atari M. Self-esteem and rhinoplasty: a case-control study. Plastic and Aesthetic Research 2016;3:111-4.

9. Dey JK, Ishii M, Phillis M, et al. Body dysmorphic disorder in a facial plastic and reconstructive surgery clinic: measur- 
ing prevalence, assessing comorbidities, and validating a feasible screening instrument. JAMA Facial Plast Surg 2015;17: 137-43.

10. American Psychiatric Association; American Psychiatric Association; Task Force on DSM-IV. Diagnostic and statistical manual of mental disorders: DSM-IV-TR. Washington, DC: American Psychiatric Association; 2000.

11. Carroll DH, Scahill L, Phillips KA. Current concepts in body dysmorphic disorder. Arch Psychiatr Nurs 2002;16:72-9.

12. Crerand CE, Phillips KA, Menard W, et al. Nonpsychiatric medical treatment of body dysmorphic disorder. Psychosomatics 2005;46:549-55.

13. Veale D, Boocock A, Gournay K, et al. Body dysmorphic disorder: a survey of fifty cases. Br J Psychiatry 1996;169:196201.

14. Veale D, Anson M, Miles S, et al. Efficacy of cognitive behaviour therapy versus anxiety management for body dysmorphic disorder: a randomised controlled trial. Psychother Psychosom 2014;83:341-53.

15. Goin JM, Goin MK. Changing the body: psychological effects of plastic surgery. Baltimore: Williams \& Wilkins; 1981.

16. Cotterill JA. Body dysmorphic disorder. Dermatol Clin 1996; 14:457-63.

17. Harris $\mathrm{D}$. The benefits and hazards of cosmetic surgery. Br J
Hosp Med 1989;41:540-5.

18. Castle DJ, Honigman RJ, Phillips KA. Does cosmetic surgery improve psychosocial wellbeing? Med J Aust 2002;176:601-4.

19. Rosen JC. Body dysmorphic disorder; assessment and treatment. In: Thompson JK, editor. Body image, eating disorders, and obesity: an integrative guide for assessment and treatment. Washington, DC: American Psychological Association; 1996. p.149-70.

20. Crerand CE, Franklin ME, Sarwer DB. Body dysmorphic disorder and cosmetic surgery. Plast Reconstr Surg 2006; 118:167e-80e.

21. Naraghi M, Atari M, Asadollahi H. When aesthetics, surgery, and psychology meet: aesthetic nasal proportions in patients having rhinoplasty and normal adults. Surg J 2016;2:e44-8.

22. Najarian B, Davoodi I. Making validation Scl25, short form Scl90. J Psychol 2001;2:136-49.

23. Gorney M, Martello J. Patient selection criteria. Clin Plast Surg 1999;26:37-40.

24. Naraghi M, Atari M. Preliminary findings on gender differences in aesthetic rhinoplasty patients: body appreciation and appearance comparisons. Otolaryngol Open J 2015;1:7-12.

25. Naraghi M, Atari M. Gender differences in aesthetic rhinoplasty patients: a study on psychopathological symptoms. Open J Med Psychol 2016;5:1-6. 